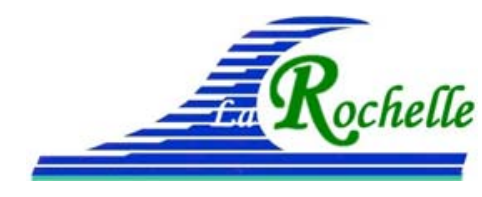

XVèmes Journées Nationales Génie Côtier - Génie Civil

La Rochelle, 29 au 31 mai 2018

DOI:10.5150/jngcgc.2018.019 (C) Editions Paralia CFL

disponible en ligne - $h$ ttp://www.paralia.fr - available online

\title{
Modélisation hydro-sédimentaire du lac de Guerlédan
}

\section{Matthis AUGER ${ }^{1}$, Tristan KEROULLE ${ }^{1}$, Lou MARILL ${ }^{1}$, Marc PEZERAT ${ }^{1}$, Arthur PRIGENT ${ }^{1}$, Valentin RUAULT ${ }^{1}$, Amandine NICOLLE ${ }^{1,2}$}

1. ENSTA-Bretagne, HOP, 2 rue François Verny, BP 92208, 29806 Brest cedex 9, France. amandine.nicolle@ensta-bretagne.fr

2. Station Biologique de Roscoff, Laboratoire Adaptation et Diversité en Milieu Marin (AD2M), UMR 7144, Place Georges Teissier, CS90074, 29688 Roscoff cedex, France.

\section{Résumé :}

Dans le cadre du projet pédagogique "les élèves hydrographes et roboticiens de l'ENSTA Bretagne explorent le lac de Guerlédan", un modèle hydro-sédimentaire haute résolution du lac de Guerlédan et de la retenue de Saint Aignan (centre Bretagne) a été développé à l'aide de TELEMAC. L'objectif de cette modélisation est de connaître la concentration maximale de sédiments sortant dans la rivière du Blavet en cas de crue extrême.

Le modèle sera confronté aux observations collectées pendant les deux semaines de terrain prévues dans le projet afin de le valider. Le modèle final pourra être exploité en mettant en place un outil pour les exploitants du lac qui permettrait, en fonction des conditions de crue, des conditions météorologiques..., de visualiser le flux sédimentaire et ainsi d'avoir un outil de gestion du barrage de Saint Aignan.

\section{Mots-clés :}

Modélisation hydro-sédimentaire, Courants, TELEMAC, Lac de Guerlédan.

\section{Introduction}

Dans le cadre du projet pédagogique "les élèves hydrographes et roboticiens de l'ENSTA Bretagne explorent le lac de Guerlédan", un modèle hydro-sédimentaire du système lac de Guerlédan - retenue de Saint Aignan haute résolution a été mis en place avec TELEMAC-3D (EDF, 2007 ; EDF, 2014). Le lac de Guerlédan est situé en Centre Bretagne. C'est un lac de barrage avec une centrale hydro-électrique gérée par EDF. En aval du barrage se trouve la retenue d'eau de Saint Aignan (voir figure 1). La rivière Le blavet alimente le lac et à la sortie de la retenue de Saint Aignan se trouve un autre barrage d'où de l'eau se déverse dans le Blavet. En amont de cette retenue se trouve une station d'eau potable, il est donc primordial de connaître la teneur en matières en suspension (MES) que peut atteindre les eaux qui sortent de la retenue de Saint Aignan. L'objectif de cette étude est donc de développer un modèle hydro-sédimentaire de ce système Lac - retenue d'eau qui couvrira les conditions hydro-sédimentaires réelles de 


\section{Thème 2 - Dynamique sédimentaire}

la zone d'étude et sera nourri des observations in situ. Les questions scientifiques traitées ici seront : la modélisation du transport de sédiments et la quantification de la concentration en sédiments qui peut être déversée dans une rivière en cas de crue.
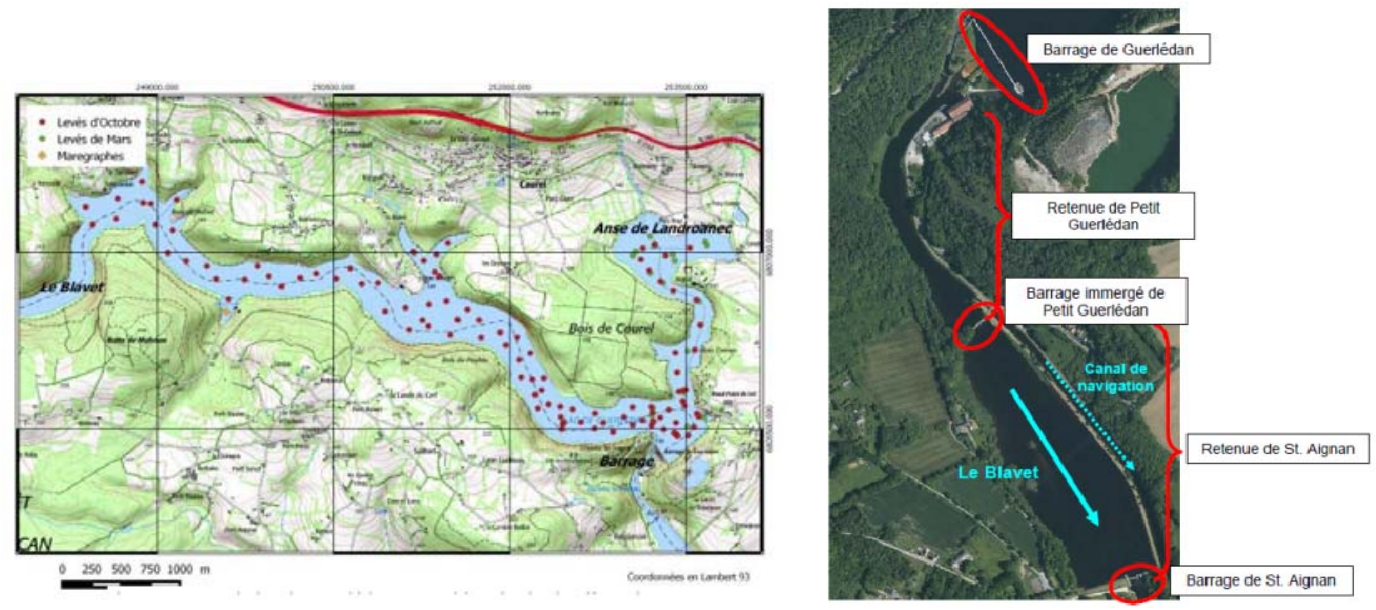

Figure 1. Zone d'étude. Lac de Guerlédan à gauche (DUYCK et al, 2017); bassin de Saint Aignan à droite, $(E D F, 2015)$.

\section{Matériel et Méthodes}

\subsection{Acquisition de données}

Différentes données ont été acquises pendant les semaines de terrain 2016, 2017 et 2018. D’autres ont été fournies par EDF (2017).

\subsubsection{Bathymétrie}

Les bathymétries 2013 du lac et de la retenue ont été fournies par EDF. Des MNT réalisés en 2017 et 2018 ont permis de calculer des différences de bathymétrie et d'estimer la quantité de sédiments mobilisables. Les mouvements sédimentaires (érosion ou dépôt) dans la retenue sont montrées par les figures 2 et 3.

Ces figures montrent que la hauteur maximale de sédiments mobilisables est de $2.7 \mathrm{~m}$. Sur le profil de la figure 3, le comblement du chenal depuis 2014 est mis en évidence.

\subsubsection{Sédiments}

Des acquisitions avec un sondeur de sédiments (ECHOES 1000) prêté par la société iXblue ont montré des zones d'accumulation de sédiments devant le barrage (figure 4). Des mesures de concentration en MES ont été réalisées en aval de Saint Aignan dans le cadre de la reprise de l'exploitation du lac de Guerlédan après la vidange de 2015 (EDF, 2016). Ces mesures effectuées du 11 janvier au 3 février 2016 (figure ${ }^{\circ} 5$ ) montrent une concentration maximale atteinte en sortie du barrage de Saint Aignan de 0.19 g/L. 


\section{XVèmes Journées Nationales Génie Côtier - Génie Civil \\ La Rochelle, 29 au 31 mai 2018}

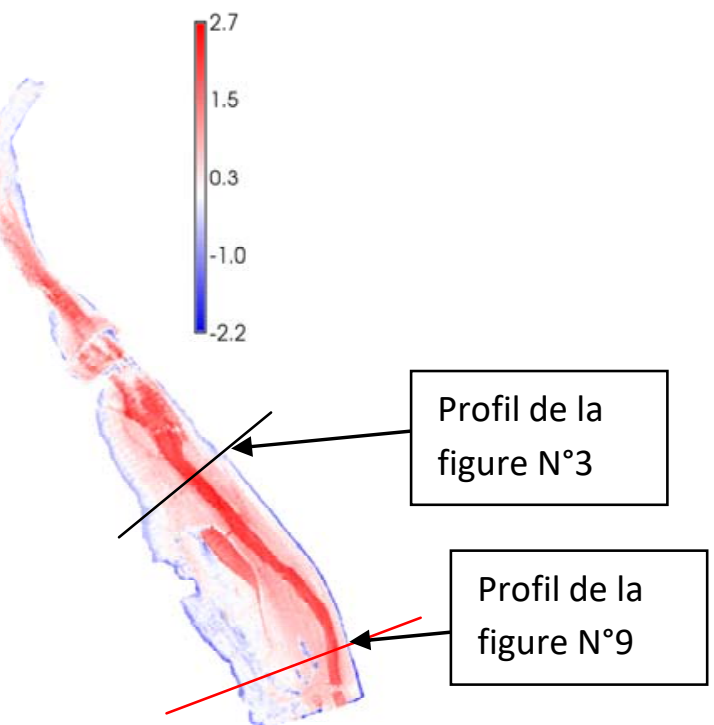

Figure 2. Différences de bathymétrie entre 2017 et 2014 (un nombre positif représente un gain en sédiments).

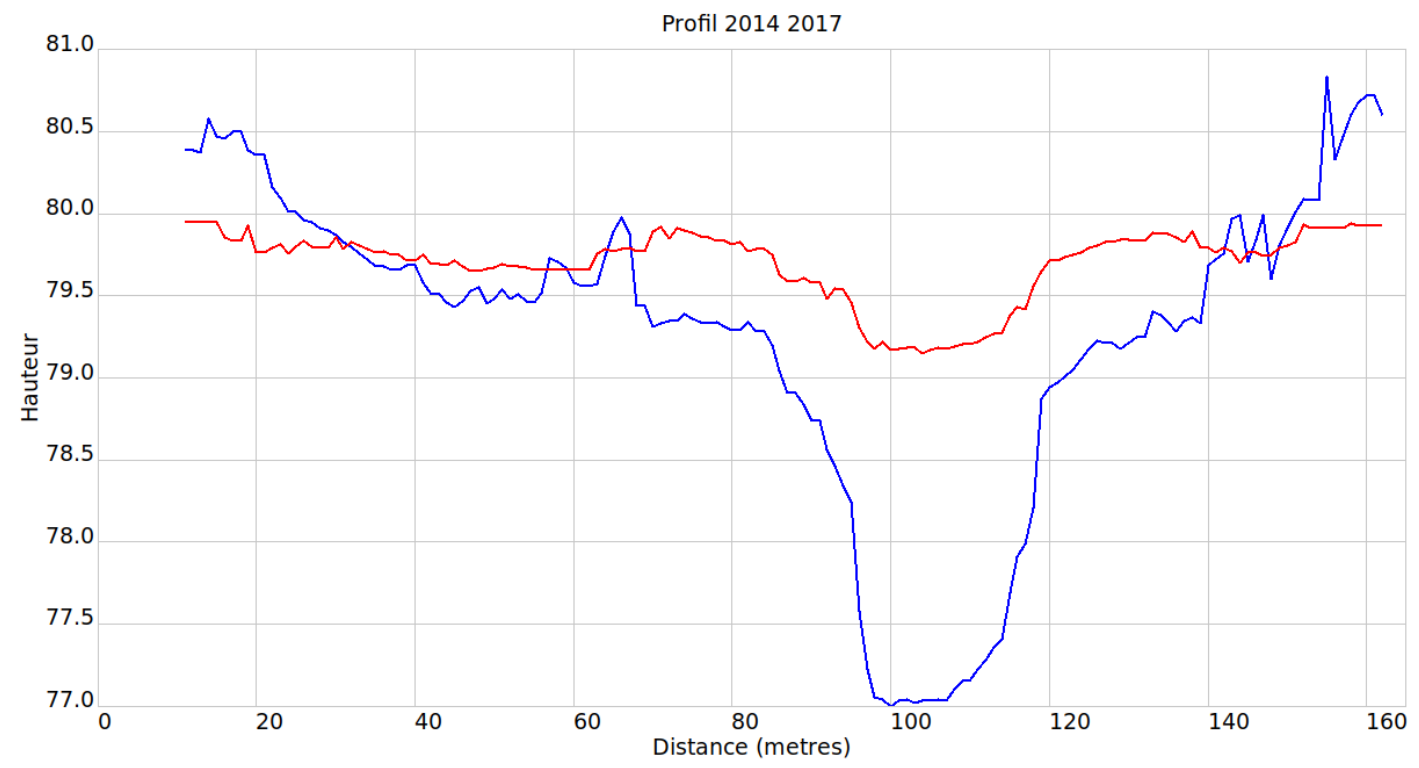

Figure 3. Profils bathymétriques de 2014(rouge) et 2017 (bleu) (au niveau du trait noir sur la fig. 2).

2.1.3 Température, chlorophylle et turbidité

Des relevés de température, chlorophylle et turbidité ont été fait en octobre 2016, février 2017, octobre 2017 et février 2018 sur le lac de Guerlédan. Pour la retenue de Saint 


\section{Thème 2 - Dynamique sédimentaire}

Aignan, nous avons des données que pour octobre 2017. Ces données nous permettent d'une part de faire un suivi environnemental du lac et d'autre part de calibrer le modèle grâce aux cartes de turbidité (figure 6).

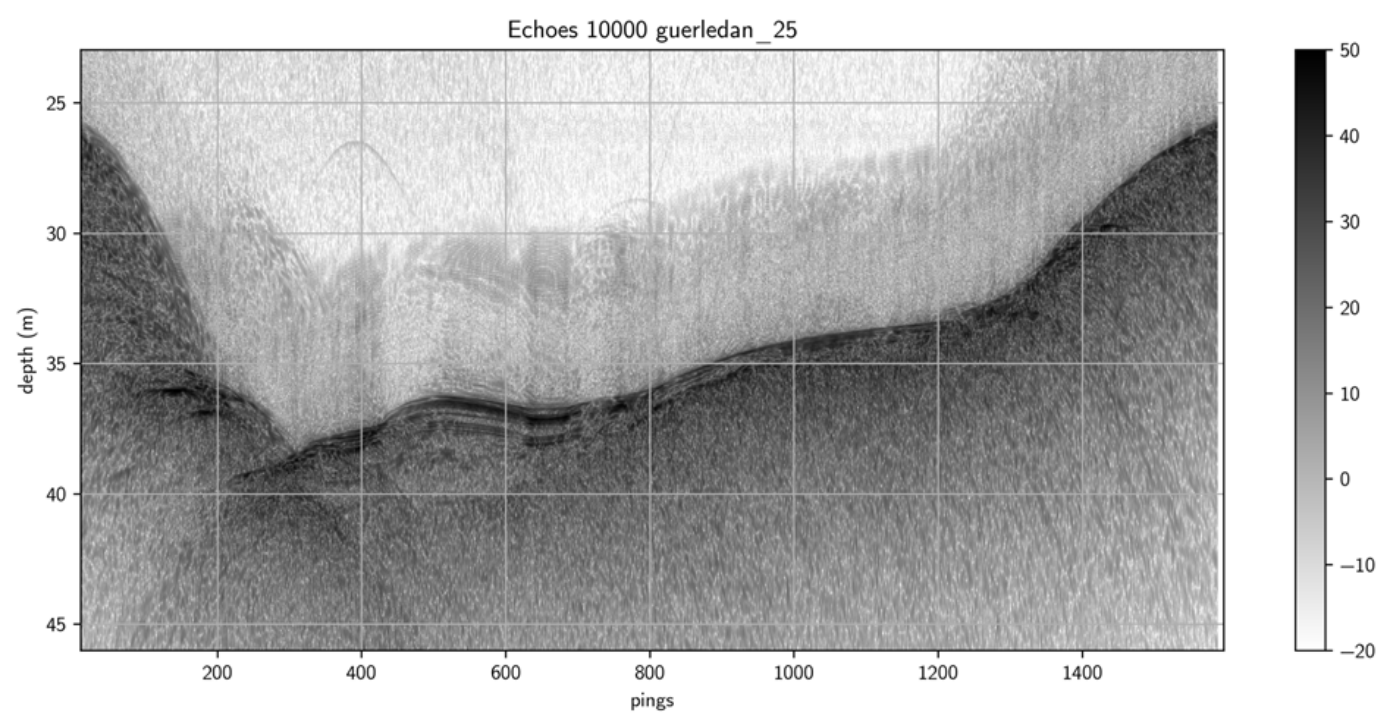

Figure 4. Données sédimentaires acquises par le sondeur sédiment ECHOES 1000 en février 2018

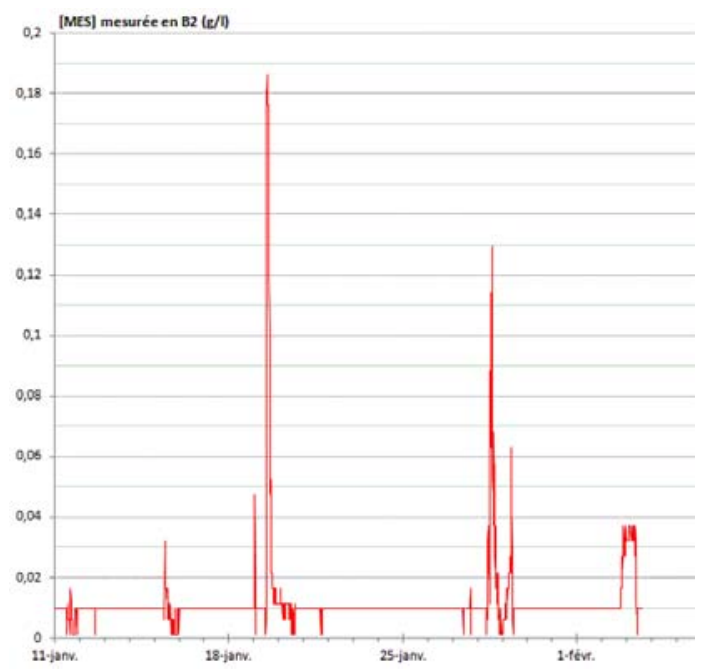

Figure 5. Données de MES mesurées en aval de Saint Aignan du 11/01/2016 au 03/02/2016 


\section{XVèmes Journées Nationales Génie Côtier - Génie Civil \\ La Rochelle, 29 au 31 mai 2018}

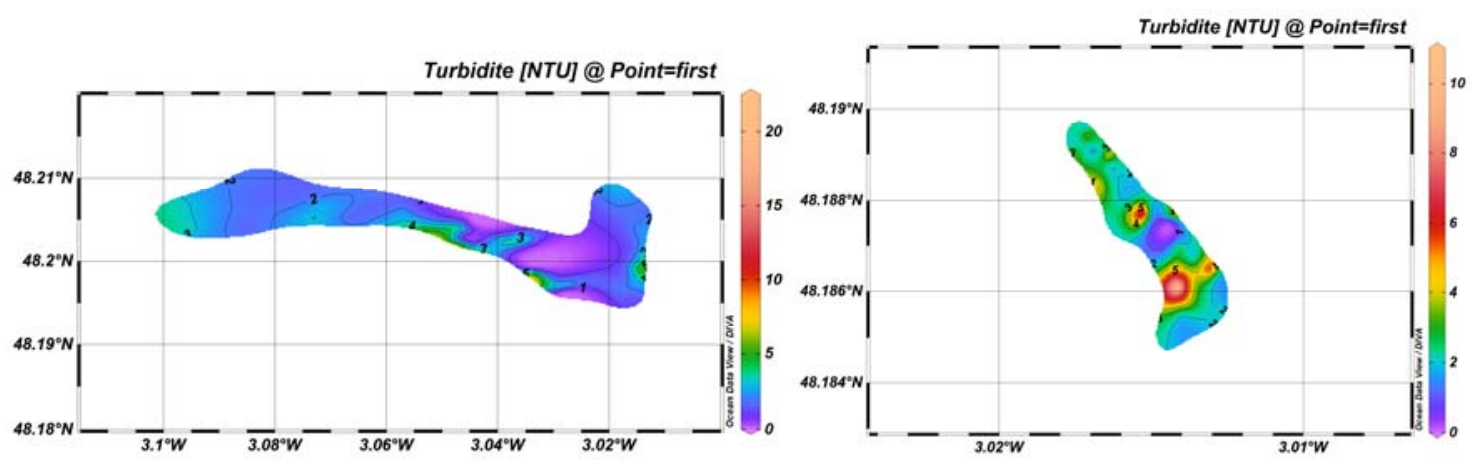

Figure 6. Carte de turbidité de surface du lac de Guerlédan (à gauche) et de la retenue de Saint Aignan (à droite)

\subsection{Description du modèle}

Le modèle développé dans cette étude est un modèle couplé TELEMAC 3D (EDF, 2007) - SISYPHE (EDF, 2014). Un premier modèle a été fait pour le lac de Guerlédan et un second pour la retenue de Saint Aignan. Les MES en sortie du lac de Guerlédan sont enregistrées pour servir de conditions limites au modèle de Saint Aignan.

Le schéma de la figure 7 montre le fonctionnement du modèle de Saint Aignan.

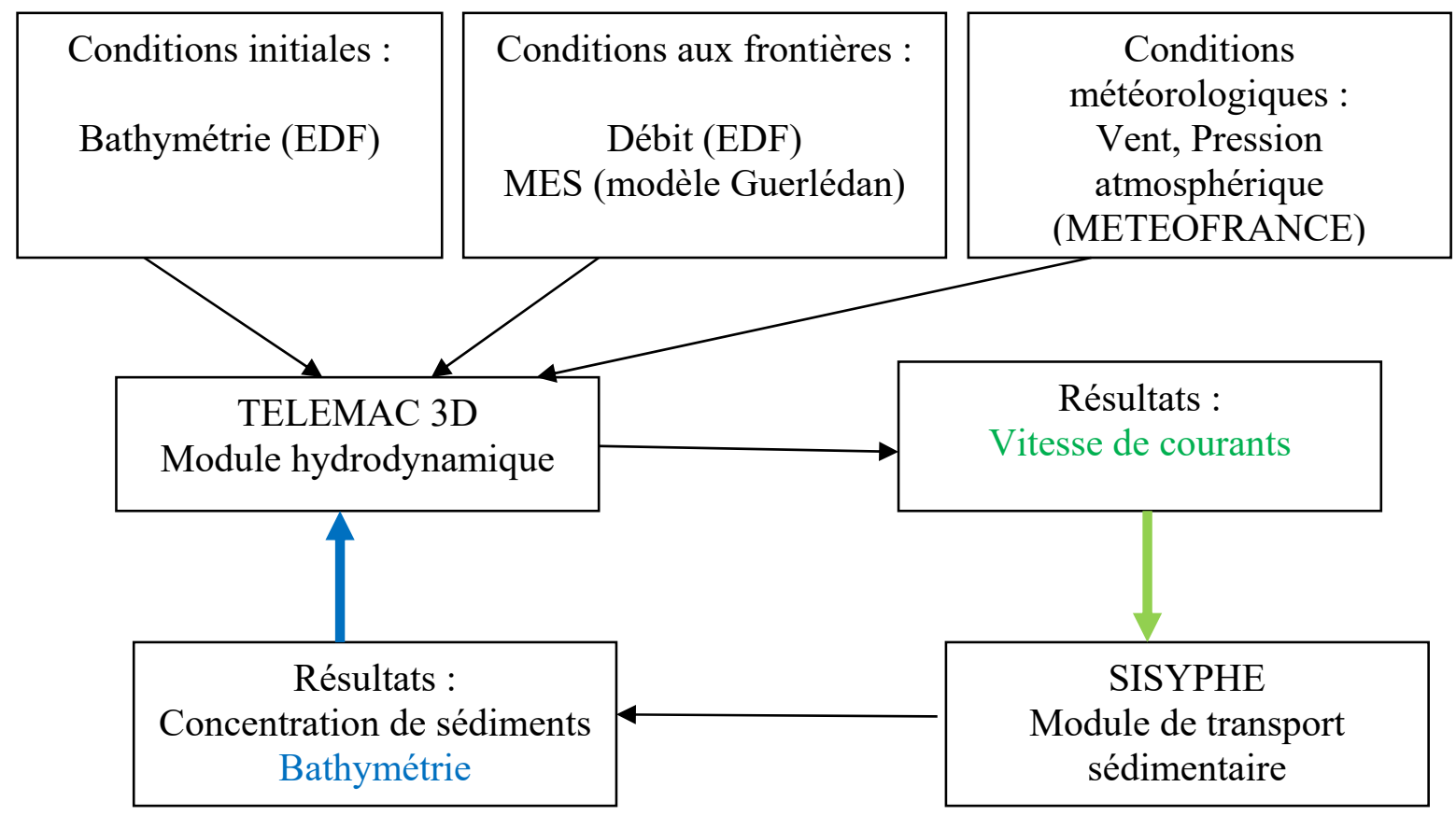

Figure 7. Schéma de fonctionnement du modèle de Saint Aignan. 


\section{Thème 2 - Dynamique sédimentaire}

\subsubsection{Maillage}

Le maillage du domaine a été construit avec le mailleur MATISSE du système TELEMAC. Il s'agit d'un maillage en éléments finis. Il est constitué de 6546 nœuds pour Saint Aignan et de 1507 nœuds pour Guerlédan. Ce nombre de nœuds de calcul est un compromis entre une résolution spatiale suffisamment fine (5 mètres de résolution spatiale pour Saint Aignan et 50 mètres pour Guerlédan) et un temps de calcul correct. Pour le maillage vertical, trois plans ont été considérés pour Saint Aignan et 10 pour Guerlédan.

\subsubsection{Conditions aux limites}

Les conditions aux limites pour le modèle de Guerlédan sont données par le débit du Blavet (SAGE, 2014-2021), et le turbinage du barrage de Guerlédan. Pour le modèle de Saint Aignan, il y a 2 types de conditions limites : le débit donné par le turbinage au niveau du barrage de Guerlédan et de celui de Saint Aignan et la concentration en MES donnée par le modèle de Guerlédan.

\subsubsection{Périodes de simulation}

Le modèle est calculé pour des conditions normales (octobre 2017) et pour des conditions de crue (février 2014). Pour ces périodes, les données de turbinage ont été fournies par EDF et les données météorologiques par METEOFRANCE.

\subsubsection{Paramétrisation du modèle}

La paramétrisation du modèle hydro-sédimentaire a été faite à l'aide des 2 documents référencés suivants : $\operatorname{EDF}(2007,2014)$. La loi permettant de modéliser le frottement sur le fond est la loi de Chézy avec un coefficient de frottement de 80 (ce qui correspond à de la vase).

Le transport sédimentaire se fait par charriage et par suspension. La hauteur maximale de sédiments mobilisables est fixée à $2.7 \mathrm{~m}$. Cette hauteur a été déduite de la différence entre les bathymétries de 2014 et 2017 (figures 2 et 3).

\section{Résultats du modèle}

\subsection{Concentration en MES}

Trois cotes initiales ont été considérées dans le modèle de Saint Aignan : $80.70 ; 79.80$ et $78.70 \mathrm{~m}$. Les résultats sont détaillés pour la cote initiale à $78.70 \mathrm{~m}$. C'est le cas où la concentration en sédiments est maximale à la sortie du modèle de Saint Aignan.

Les simulations ont été faites en période normale et en période de crue. Les résultats montrés ici correspondent à un cas où il y aurait 3 crues successives. L'objectif étant de connaître la concentration maximale de sédiments qui pourrait être déversée dans le Blavet à la sortie du barrage de Saint Aignan. 


\section{XVèmes Journées Nationales Génie Côtier - Génie Civil \\ La Rochelle, 29 au 31 mai 2018}

Le graphique suivant (figure 8 en haut) montre l'évolution de la cote au niveau du barrage de Saint Aignan et du débit entrant par le barrage de Guerlédan. L'évolution de la concentration en sédiments au niveau de la sortie du barrage de Saint Aignan est représentée sur la figure 8 (en bas).

La concentration en sédiments maximale sortante modélisée ne dépasse pas $0.13 \mathrm{~g} / \mathrm{L}$. Elle est obtenue dès le début de la simulation.

Le premier pic de concentration $(0.127 \mathrm{~g} / \mathrm{L})$ apparaît au début de la simulation. Il est corrélé avec une chute de la cote à $77.9 \mathrm{~m}$.

Un deuxième pic $(0.03 \mathrm{~g} / \mathrm{L})$ apparait pour un débit entrant supérieur à $80 \mathrm{~m}^{3} / \mathrm{s}$. La cote remonte à $78 \mathrm{~m}$.

Un troisième pic $(0.03 \mathrm{~g} / \mathrm{L})$ est obtenu au moment du pic de crue $\left(140 \mathrm{~m}^{3} / \mathrm{s}\right)$. La cote baisse à $77.8 \mathrm{~m}$.

Un quatrième pic $(0.04 \mathrm{~g} / \mathrm{L})$ est obtenu au moment du pic de la $2^{\text {ème }}$ crue. Le sédiment mobilisable est en grande partie évacué dès la première crue.

Les résultats sont cohérents avec les observations faites par EDF (2016). La différence entre la concentration maximale observée au moment de la reprise de l'exploitation et celle modélisée vient du fait que dans le modèle, nous sommes partis des données de 2017 (1 an après la reprise de l'exploitation) et donc la quantité de sédiments mobilisables est moins importante.

\subsection{Creusement du chenal}

Des profils bathymétriques ont été faits après le passage des trois crues. Le résultat est représenté sur la figure 9.

Le creusement du chenal se fait sur une largeur conséquente. Il faut voir que cette figure est obtenue après passage de trois crues successives qui laissent peu de possibilités pour le sédiment de se redéposer. Ce creusement large et localisé est dû aux vitesses très importantes au moment du pic de crue (Figure 10). Le courant induit par le fort débit se propage en ligne droite et n'impacte pas les zones latérales. Le fait que le creusement du canal soit décalé par rapport à ce que l'on peut voir sur la bathymétrie de 2017 est lié à la localisation des évacuateurs. Les évacuateurs du barrage sont situés surtout sur un côté du barrage alors que dans le modèle, les évacuateurs virtuels sont répartis sur toute la largeur. 
Thème 2 - Dynamique sédimentaire
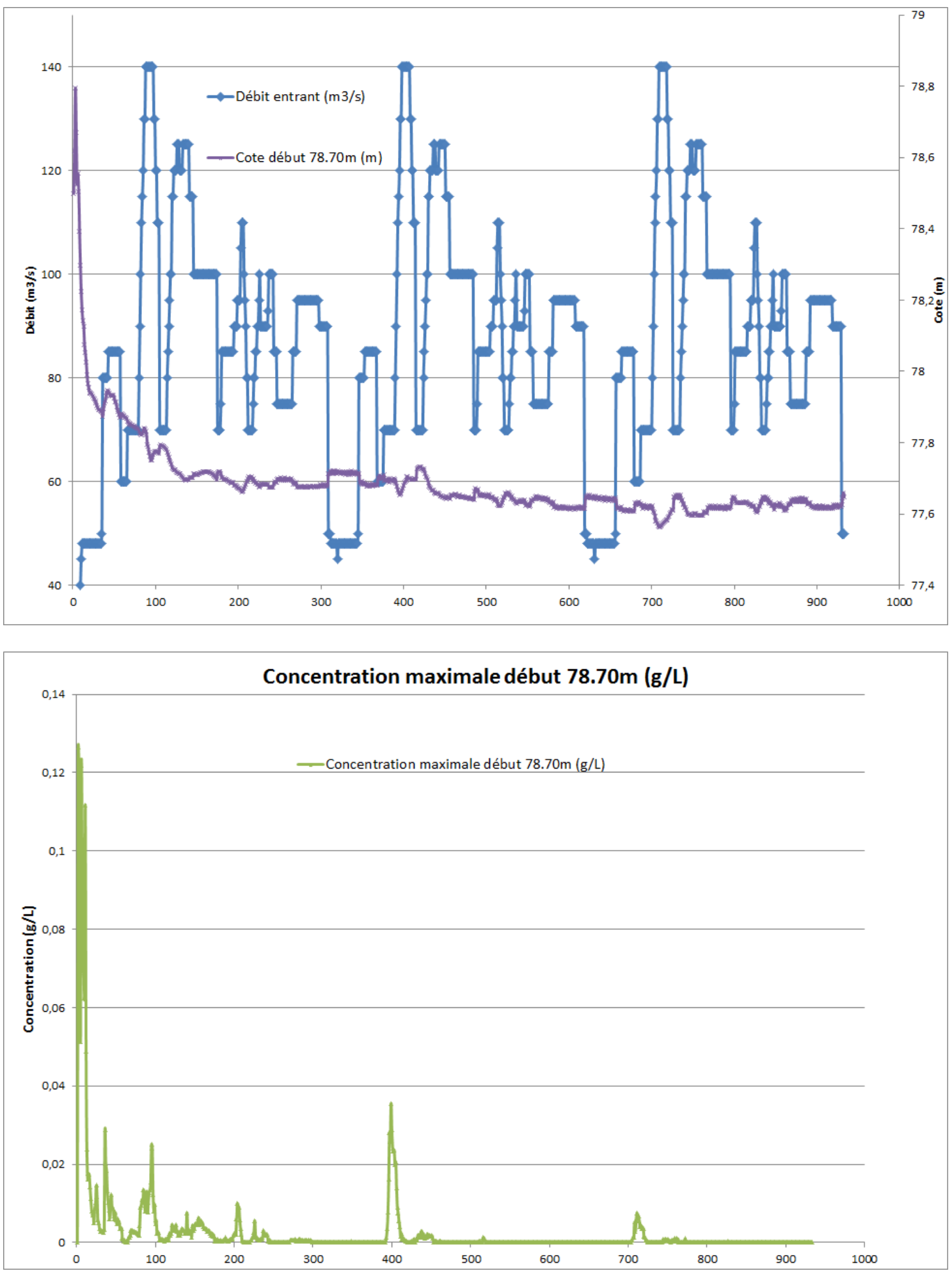

Figure 8. Débit à l'entrée du modèle et cote au niveau du barrage de Saint Aignan (en haut) et évolution de la concentration en sédiments en sortie du modèle (en bas). 


\section{XVèmes Journées Nationales Génie Côtier - Génie Civil \\ La Rochelle, 29 au 31 mai 2018}

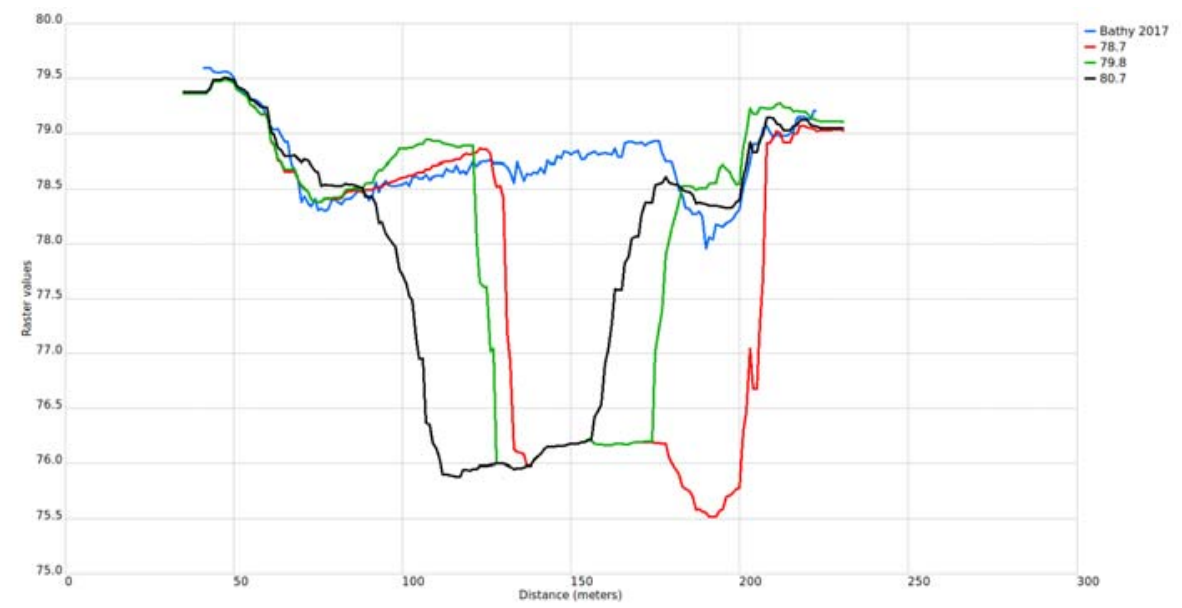

Figure 9. Profils bathymétriques après le passage des 3 crues pour les 3 cas simulés (au niveau du trait rouge sur la figure 2).

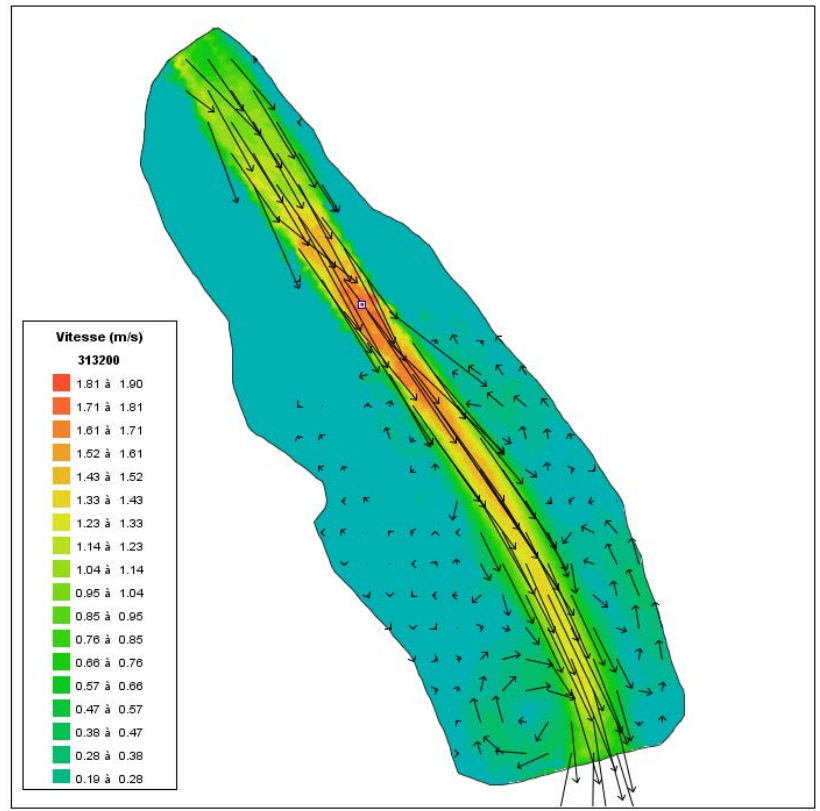

Figure 10. Champs de vitesse issu du modèle à la pointe de la crue.

\section{Conclusion}

Un modèle hydro-sédimentaire tridimensionnel a été mis en place pour le système lac de Guerlédan / bassin de Saint Aignan à l'aide de TELEMAC.

Le modèle a été paramétré grâce à des données acquises sur le terrain et des données fournies par EDF et METEOFRANCE.

Les résultats de la modélisation montrent qu'après 3 crues de type de celle de février 2014, la concentration maximale de sédiments en sortie de Saint Aignan est de $0.13 \mathrm{~g} / \mathrm{L}$ 


\section{Thème 2 - Dynamique sédimentaire}

et le volume total de sédiments perdu est de $54056 \mathrm{~m}^{3}$. Il semble donc que, même pour un scénario catastrophe, le seuil maximal autorisé de $0.3 \mathrm{~g} / \mathrm{L}$ ne soit jamais atteint.

\section{Références}

EDF (2007). Logiciel TELEMAC-3D Version 5.8 - Manuel de l'utilisateur. Décembre 2007.

EDF (2014). Logiciel SISYPHE Version 6.3 - Manuel de l'utilisateur. Janvier 2014.

EDF (2015). Reprise d'exploitation de l'usine de Guerlédan: modélisation sédimentaire avec le logiciel COURLIS de l'impact sur la concentration en MES à l'aval du bassin de St Aignan. Rapport IH.GUERL-QUAL.GC-HY.00001.A.

EDF (2016). Vidange 2015. Bilan des dépôts sédimentaires à St-Aignan et analyse du risuqe de remobilisation. Rapport IH-GUERL-VDGE.ENV.00013 A.

EDF (2017). Bathymétrie des bassins de Saint Aignan en avril 2017. Rapport H44200961-2017-000120.

DUYCK E., GERMAIN C., INGOUF C. (2017). Surveillance environnementale du lac de Guerlédan. Rapport de projet élèves HYO $3^{\text {ème }}$ année ENSTA Bretagne.

SAGE (2014-2021). Schéma d'Aménagement et de Gestion des Eaux, SAGE Blavet 2014-2021. URL : http://www.sage-blavet.fr/zoom-sur/le-barrage-de-guerledan 